\title{
2 Diagnostic Imaging in Pediatric Chronic Rhinosinusitis: A Guide for Clinicians
}

\author{
3 Giorgio Conte ${ }^{1}$, Luca Caschera ${ }^{2}$, Sandro Magnani ${ }^{2}$, Claudia Cinnante ${ }^{1}$, Fabio Maria Triulzi ${ }^{\text {, }}$ \\ 4 Lorenzo Pignataro ${ }^{3,4}$ and Sara Torretta ${ }^{3,4}$ \\ 1 Fondazione IRCCS Ca'Granda Ospedale Maggiore Policlinico, Department of Neuroradiology, Milan, Italy \\ 2 Postgraduation School in Radiodiagnostics, Università degli Studi di Milano, Milan, Italy \\ 3 Fondazione IRCCS Ca'Granda Granda Ospedale Maggiore Policlinico, Department of \\ Otorhinolaringology, Milan, Italy \\ 4 Università Statale di Milano, Department Clinical Sciences and Community Health, Milan Italy \\ * Correspondence: giorgioconte.unimed@gmail.com; tel: +39-0255035160 (G.C).
}

Abstract: Pediatric rhinosinusitis are common encountered disorders caused by different pathogenic factors or predisposing conditions with a proportion of them progressing to chronicity with a meaningful impact on quality of life and related adverse effects. A deep understanding of pathophysiology, disease course and prognosis are the fundamental building block to an appropriate management of rhinosinusitis in order to avoid risks related to complications. This review gives concise answers to clinicians who want to know "when do we need imaging?" "what do we aspect from imaging?" and "what are the appropriate imaging modalities in different settings?" in pediatric patients in order to increase the appropriateness of imaging examinations.

Keywords: rhinosinusitis; pediatric chronic rhinosinusitis; computed tomography; magnetic resonance imaging

\section{Introduction}

Rhinosinusitis is a common disorder related to inflammation of the paranasal and nasal sinus mucosae. Specifically rhinosinusitis is common in children, caused by different pathogenic factors or predisposing conditions. The diagnosis in most cases should be made on clinical grounds alone. It affects 31 million individuals per year and the prevalence is higher in children than adults (32\% in children under 6 years of age), with $5-10 \%$ of all upper respiratory tract infections being complicated by rhinosinusitis, some of them developing into a chronic form [1]. Besides a deep knowledge of pathophysiology, associated/predisposing conditions, disease course and prognosis, an appropriate diagnostic and therapeutic management of pediatric chronic rhinosinusitis improves patients' outcome by avoiding risks related to complications. This review aims to answer clinicians' questions, like "when do we need imaging?", "what do we aspect from imaging?" and "what are the appropriate imaging modalities in different settings?" in order to increase the appropriateness of imaging studies in pediatric patients avoiding the unnecessary radiation exposure.

\section{Anatomy and Pathophysiology}

Paranasal sinuses begin to develop during fetal life and progress to postnatal period, typically becoming fully developed by age 12-14 years and reaching definitive size by 19 years. During fetal life and childhood paranasal sinuses evolve and redefine continuously their boundaries inside the host bony structures of the splanchnocranium: the ethmoid and maxillary sinuses are the first to appear usually on the 3rd month of gestation, being normally present at birth with a subsequent progressive growth, reaching their adult size by the age of 10 years. The sphenoid sinus usually develops before 3 years of age and its size reaches the maximum at around 12-14 years of age [2]. The frontal sinus is the last to develop at around 5 years of age reaching its complete pneumatization in the late adolescence [3]. Based on age-related anatomic differences of paranasal 
sinuses, treatment of chronic rhinosinusitis in children below 12 years is inherently different from that of older children [4].

The nasal sinuses are lined by mucosal tissue consisting of mucus goblet cells interspersed in a pseudo-stratified ciliated columnar epithelium. The composite term "rhino+sinusitis" better defines the affection since sinusitis is almost always associated with or preceded by signs and symptoms of rhinitis. This is due to the fact that the nasopharyngeal mucosal lining is continuous with the paranasal sinus mucosae and any process that affects the nasal mucosae may spread to the sinus cavities. The secreted mucous and glicoproteins and the generated active mucociliary transport are key to maintain a healthy sinus environment by inhibiting the ability of bacteria to colonize the epithelium and by removing dust and microparticles. Furthermore a number of local and systemic factors contribute to the development of sinusitis like first of all anatomic or pathologic conditions that interferes with the normal sinus drainage pathways by obstructing sinuses outflow; this process generates a cascade of events starting from the reduction of oxygen supply to the sinus, ciliary and mucous glands disfunction with subsequent vasodilation of local vasculature, mucosal swelling and stagnation of fluid that may become acutely or chronically infected [4, 5]. In contrast to the acute form, the chronic rhinosinusitis may recognize multiple predisposing factors, from genetic to chronically obstructive and allergic causes. Several studies have demonstrated a genetic predisposition to chronic rhinosinusitis [6] highlighting potassium channel and other ion transport channel disorders [7], primary immunodeficiencies, Kartagener's syndrome and cystic fibrosis as strongly associated with it [8]. Allergic rhinitis [9] and asthma [10] are also frequently associated to chronic rhinosinusitis.

\section{Definition}

According to American Academy of Pediatrics (AAP), based on the duration of symptoms, rhinosinusitis is classified into acute, subacute and chronic forms. This classification is important because of inherently differences in diagnostic methods, treatment and follow up strategies and possible complications.

\subsection{Acute rhinosinusitis}

Acute rhinosinusitis is defined as a persistent illness with nasal discharge of any quality and/or daytime cough lasting for $>10$ days without improvement, a worsening clinical course, a severe onset of symptoms with concurrent fever (temperature $\geq 39^{\circ} \mathrm{C}$ ), and purulent nasal discharge for at least 3 consecutive days [11].

\subsection{Subacute rhinosinusitis}

Subacute rhinosinusitis is defined as a sinusitis that lasts between 30 and 90 days and whose symptoms resolve completely. Recurrent acute bacterial sinusitis is defined by episodes lasting $<30$ days each and separated by intervals of at least 10 asymptomatic days [12].

\subsection{Chronic rhinosinusitis}

Chronic rhinosinusitis is defined as at least 90 continuous days of two or more symptoms: purulent rhinorrhea, nasal obstruction, facial pressure/pain, cough and either endoscopic signs of mucosal edema, purulent drainage, nasal polyposis and/or computed tomography scan showing mucosal changes within the ostiomeatal complex and/or sinuses in a pediatric patient aged 18 years or younger. This definition of chronic sinusitis in pediatric patients is similar to what has been accepted in adults [13].

\section{Diagnostic imaging: when, why, how}

Rhinosinusitis doesn't have always a straightforward indication to Radiology particularly in the acute forms in children under six years old where the initial diagnostic work-up should be 
clinical [14]. In cases of uncomplicated acute rhinosinusitis the clinical examination alone should guide the diagnosis and prompt treatment decisions. In this context even if it is difficult to clinically differentiate between viral and bacterial sinusitis (often associated), imaging isn't still recommended as it does not change therapeutic management. Moreover imaging abnormalities, such as paranasal sinus opacification on computed tomography (CT), are not sufficient to reach the diagnosis since they could be also detected in healthy children undergoing imaging examinations for other reasons $[15,16]$. CT abnormalities may be demonstrated in up to $87 \%$ of subjects recovering from a cold [12], MRI abnormalities in $68 \%$ of symptomatic children with upper respiratory tract infection and $42 \%$ of healthy children [15].

Nowadays plain X-rays and ultrasonography are not recommended in the evaluation of sinusitis. CT is considered the gold standard imaging technique and the latest generation CT scanners may help reducing the dose using new algorithms of image reconstruction and processing [17]. The innovative use of cone beam CT guarantees a reduction of up to $30 \%$ of the effective dose in comparison to Multiple Detector CT (MDCT) and the effective radiation dose can be lowered to a level comparable to that used for standard radiographic images [12, 18].

According to a consensus statement on the appropriate use of CT for paranasal sinuses disease [18], CT should be performed in the following scenarios:

- in children that has failed appropriate medical management;

- in patients who have been treated repeatedly with antibiotics and other medications for presumed sinusitis, with persistent complaints post-treatment, but no clinical evidence of sinusitis, to confirm that the problem is not due to sinonasal pathology and avoid continued inappropriate antibiotic administration;

- for recurrent acute and chronic sinusitis;

- for suspected tumor, complications from sinusitis, and prior to sinus surgery [12, 18];

- in patients with Cerebral Spinal Fluid rhinorrhea (suspected or confirmed, spontaneous or iatrogenic).

In the setting of cystic fibrosis (CF), the altered ion transport across cell membranes lead to the formation of more viscous respiratory secretions with impaired normal mucociliary transport and subsequent sinonasal inflammation and obstructive polyposis. Nowadays in the era of advanced medical therapies the life expectancy of CF patients has increased dramatically; this has resulted in repeated follow up CT scan during their lifetime and subsequent increased exposure to ionizing radiation especially in young children who are particularly susceptible to potential radiation-induced carcinogenesis. In a recent study on 202 pediatric patients with CF who underwent endoscopic sinus surgery (ESS) a total of 1718 CT scan was reported; ninety CT scans $(10.8 \%)$ didn't led to change in management and no significant differences in Lund Mackay (LM) scores were reported either between patients admitted to the hospital/prescribed antibiotics or those who were not, and following ESS. Effort should be made to reduce radiation exposure in patients with $\mathrm{CF}$ by limiting $\mathrm{CT}$ scans to the preoperative management or for evaluation of potential sinus and intracranial complications [19].

Magnetic resonance imaging (MRI) with conventional and advanced sequences (such as MR angiography), considering its superb contrast resolution and its ability to detect vascular complications is strongly encouraged when intracranial or intraorbital or vascular extension are suspected. MR angiography should be combined with the standard MRI protocol in order to not miss other potential complications, with the disadvantage of increasing the scan time. Arterial MR angiography is always performed without contrast-agent administration. Venous MR angiography could be performed both with and without intravenous contrast-agent administration, however non-contrast technique should be recommended in those patients with contraindication to the administration or when the contrast-agent is not required for the complementary standard MR examination [12].

\section{Imaging Features}


Varying degrees of mucosal swelling and sinus opacification are key imaging sign of chronic rhinosinusitis. Other frequent characteristics are hyperostosis of the sinus walls (mucoperiosteal reaction) and thickening of the mucosal lining. Specifically the presence of calcifications may suggest the diagnosis of fungal sinus infection [20].

Chronic forms of sinusitis may be associated with intracranial and intraorbital complications in addition to bone involvement such as optic neuritis, orbital and periorbital cellulitis, orbital and subperiosteal abscess, meningitis, subdural and epidural empyema, brain abscess and venous sinus thrombosis, maxillary osteitis and frontal osteitis (Pott puffy tumor). In such cases and in those associated with cranial nerve palsies or mental status impairment, contrast enhanced CT is recommended. The frontal skull appears to be particularly vulnerable to spread of infection, likely because of its rich network of diploic veins [21]. Some recent studies have tried to estimate the prognosis and the need of surgical management for subperiosteal orbital abscess through CT 3D-sotfware reconstruction and calculation of abscess volume and dimensions. This studies demonstrated that the greater the volume of the abscess, the higher the necessity of surgical management, but the cut-off are different between the studies: some observed that an abscess greater than $500 \mathrm{~mm}^{3}$ was associated with $100 \%$ of surgical management [22, 23], others showed that patients with an abscess width greater or equal to $1 \mathrm{~cm}$ were usually managed surgically [24, 25].

\subsection{MRI}

MRI may require sedation in young children. Studies have shown that MRI is significantly more accurate than CT (97\% versus $87 \%$ ) in diagnosing intracranial complications and moreover diffusion-weighted imaging (DWI) can localize or confirm the presence of purulent material as it typically shows a restricted diffusion [11, 12]. Although MRI is also more sensitive than CT in diagnosing the presence of osteomyelitis, it does not demonstrate the bony detail of the osteomeatal complex well and is less sensitive to bony erosions, making it less well suited than CT to evaluate for underlying anatomic abnormalities that may predispose to chronic sinusitis.

Accordingly, the most recent appropriateness criteria endorse both MRI with contrast and contrast-enhanced CT as complementary examinations when evaluating potential complications of sinusitis [12]. Gadolinium injection can improve the detection of disease extent and its eventual diffusion beyond the paranasal sinuses either in the orbits and/or in the intracranial compartment [26].

\subsection{Key imaging points:}

- Varying degrees of smooth or irregular mucosal swelling due to edema and secretion

- Sclerotic and thickened sinus walls

- Dystrophic micro or gross calcifications suggest a chronic fungal sinus infection

- In chronic fungal sinusitis MRI shows a decreased signal intensity on T1-weighted images and a markedly decreased signal intensity on T2-weighted images.

- T1-weighted MRI: low to intermediate signal intensity of the sinus mucosal lining

- T2-weighted MRI: usually inflamed mucosa appears hyperintense while sclerosis and fibrous tissue shows low T2 signal.

- MRI is able to differentiate sinus opacification caused by inflammation from neoplasms by distinguishing soft tissue from dense secretions; while secretions are T2 hyperintense, neoplasms present a more intermediate iso-hyperintense T2 signal [27].

- CT angiography and MRI with conventional and advanced imaging sequences should be considered in case of suspected intraorbital, vascular and/or intracranial disease extension in order to rule out possible life threatening complications. 


\section{Conclusion}

The continuous update of guidelines in addition to new CT algorithms for dose reduction and the large-scale availability of MRI scanners allows to reduce and limits the radiation burden in the pediatric population that need to be evaluated for chronic sinusitis and the related complications. Plain-film radiography doesn't have anymore a role between the diagnostic tools for investigating sinuses diseases due to the lack of diagnostic power and the inability to adequately show disease processes. MRI is characterized by higher capacities in differentiating inflammatory conditions from neoplastic processes and provides superb accuracy in showing the presence of intracranial or infraorbital complication that could be life-threatening for the child. CT scanning allows a perfect definition of sinonasal bony anatomy and CT scans should be obtained before functional ESS as bony landmarks depicted could serve as a "road map" for surgery. According to the most recent guidelines both CT and MRI are nowadays considered fundamental instruments in the evaluation of pediatric chronic rhinosinusitis.

Author Contributions: “conceptualization, G.C., L.C., S.M., C.C., F.M.T., L.P., S.T.; writing-review and editing G.C., L.C., S.M., C.C., F.M.T., L.P., S.T..; supervision, G.C., L.P.;

Funding: This research received no external funding.

Conflicts of Interest: The authors declare no conflict of interest.

\section{References}

1. Anzai Y.; Paladin A. Diagnostic imaging in 2009: update on evidence-based practice of pediatric imaging. What is the role of imaging in sinusitis? Pediatr Radiol 2009, 39 (Suppl 2):S239-S241 DOI 10.1007/s00247-008-1128-y

2. Hamberger C., Hammer G.; Norlen G.; Sjogren B. Transantrosphenoidal hypophysectomy. Arch Otolaryngol. 1961 Jul;74:2-8.

3. DeMuri G.; Wald ER. Acute bacterial sinusitis in children. Pediatrics in review/American Academy of Pediatrics. 2013; 34(10):429-37. doi:10.1542/pir.34-10-429.

4. Badr T.; Gaffin J.; Phipatanakul W. Pediatric Rhinosinusitis. Curr Treat Options Allergy 2016 September; 3(3): 268-281. doi:10.1007/s40521-016-0096-y.

5. Moore BM.; Blumberg K.; Laguna TA.; Liu M.; Zielinski EE.; Kurachek SC. Incidental sinusitis in a pediatric intensive care unit. Pediatric critical care medicine. 2012; 13(2):e64-8. doi:10.1097/PCC.0b013e31820ac3f5.

6. Orb Q.; Curtin K.; Oakley GM.; Wong J.; Meier J.; Orlandi RR et al. Familial risk of pediatric chronic rhinosinusitis. The Laryngoscope. 2015 doi:10.1002/lary.25469.

7. Purkey MT.; Li J.; Mentch F.: Grant SF.; Desrosiers M.; Hakonarson H. et al. Genetic variation in genes encoding airway epithelial potassium channels is associated with chronic rhinosinusitis in a pediatric population. PloS one. 2014; 9(3):e89329. doi:10.1371/journal.pone.0089329.

8. Min JY.; Tan BK. Risk factors for chronic rhinosinusitis. Curr Opin Allergy Clin Immunol.. 2015; 15(1):1-13. doi:10.1097/aci.0000000000000128.

9. Sedaghat AR.; Phipatanakul W.; Cunningham MJ. Prevalence of and associations with allergic rhinitis in children with chronic rhinosinusitis. Int $J$ Pediatr Otorhinolaryngol. 2014; 78(2):343-7. doi:10.1016/j.jporl.2013.12.006.

10. Anfuso A.; Ramadan H.; Terrell A.; Demirdag Y.; Walton C.; Skoner DP. et al. Sinus and adenoid inflammation in children with chronic rhinosinusitis and asthma. Ann Allergy Asthma Immunol. 2015; 114(2):103-10. doi:10.1016/j.anai.2014.10.024.

11. Wald ER.; Applegate KE.; Bordley C. et al. Clinical practice guideline for the diagnosis and management of acute bacterial sinusitis in children aged 1 to 18 years. Pediatrics 2013;132:e262-80.

12. Expert Panel on Pediatric Imaging:, Tekes A.; Palasis S.; Durand DJ. Et Al. ACR Appropriateness Criteriaß Sinusitis-Child. J Am Coll Radiol. 2018 Nov;15(11S):S403-S412. doi: 10.1016/j.jacr.2018.09.029.

13. Brietzke E.; Shin J.; Choi S.; Lee T.; Parikh R.; Pena M. et Al. Clinical Consensus Statement: Pediatric Chronic Rhinosinusitis. Otolaryngology - Head and Neck Surgery 2014 151: 542 DOI: $10.1177 / 0194599814549302$ 
14. American Academy of Pediatrics. Subcommittee on Management of Sinusitis and Committee on Quality Improvement. Clinical practice guideline: management of sinusitis. Pediatrics 2001;108:798-808.

15. Brook I. Acute sinusitis in children. Pediatr Clin North Am 2013;60:409-24.

16. Glasier CM, Mallory GB, Jr., Steele RW. Significance of opacification of the maxillary and ethmoid sinuses in infants. J Pediatr 1989;114:45-50

17. Triulzi F.; Zirpoli S. Imaging techniques in the diagnosis and management of rhinosinusitis in children. Pediatr Allergy Immunol 2007: 18 (Suppl. 18): 46-49 DOI: 10.1111/j.1399-3038.2007.00633.x

18. Setzen G.; Ferguson BJ.; Han JK. Clinical consensus statement: appropriate use of computed tomography for paranasal sinus disease. Otolaryngol Head Neck Surg. 2012 Nov;147(5):808-16. doi: 10.1177/0194599812463848. PMID: 23054429

19. Gergin O, Kawai K, MacDougall RD. Sinus Computed Tomography Imaging in Pediatric Cystic Fibrosis: Added Value? Otolaryngol Head Neck Surg. 2016 Jul;155(1):160-5.

20. Yoon JH.; Na DG.; Byun HS et Al. Calcification in chronic maxillary sinusitis: comparison of CT findings with histopathologic results. AJNR Am J Neuroradiol. 1999;20 (4): 571-4.

21. Germiller JA, Monin DL, Sparano AM, Tom LW. Intracranial complications of sinusitis in children and adolescents and their outcomes. Arch Otolaryngol Head Neck Surg. 2006 Sep;132(9):969-76.

22. Tabarino F, Elmaleh-Bergès M, Quesnel S, Lorrot M, Van Den Abbeele T, Teissier N. Subperiosteal orbital abscess: volumetric criteria for surgical drainage. Int J Pediatr Otorhinolaryngol. 2015 Feb;79(2):131-5. doi: 10.1016/j.ijporl.2014.11.021

23. Gavriel H, Yeheskeli E, Aviram E, Yehoshua L, Eviatar E. Dimension of subperiosteal orbital abscess as an indication for surgical management in children. Otolaryngol Head Neck Surg. 2011 Nov;145(5):823-7. doi: 10.1177/0194599811416559.

24. Reza R, Caroline D. Robson, Robert A. Petersen et al. Management of Orbital Subperiosteal Abscess in Children. Arch Otolaryngol Head Neck Surg. 2001;127(3):281-286.

25. Todman MS, Enzer YR. Medical management versus surgical intervention of pediatric orbital cellulitis: the importance of subperiosteal abscess volume as a new criterion. Ophthalmic Plast Reconstr Surg. 2011 Jul-Aug;27(4):255-9.

26. Leo G, Incorvaia C, Masieri S, Triulzi F. Imaging criteria for diagnosis of chronic rhinosinusitis in children. Eur Ann Allergy Clin Immunol. 2010 Dec;42(6):199-204.

27. Branstetter BF, Weissman JL. Role of MR and CT in the paranasal sinuses. Otolaryngol Clin North Am 2005;38:1279-99. 